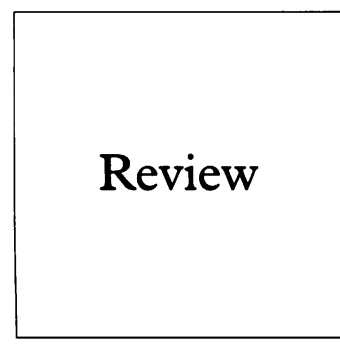

\title{
From human immunodeficiency virus (HIV) infection of the brain to dementia
}

\author{
G Trillo-Pazos, I P Everall
}

Human immunodeficiency virus (HIV) can cause both primary and secondary brain diseases. Numerous neuropathological studies have shown that up to $90 \%$ of patients with acquired immune deficiency syndrome (AIDS) have lesions in the nervous system. In this review, we discuss the entry of HIV into the brain, the general features of HIV associated neuropathology, the role of different brain cells in HIV mediated neuronal damage, and the putative molecular mechanisms involved. We conclude by correlating which factors might be important in the development of HIV associated dementia.

(Genitourin Med 1997;73:343-347)

Keywords: HIV; AIDS; brain; dementia

HIV entry into the brain

It is unclear whether human immunodeficiency virus (HIV) enters the brain soon after the initial systemic infection or later during the course of the asymptomatic phase of disease. ${ }^{1-3}$ The brain is considered to be an "immune privileged site" as the blood-brain barrier (BBB) prevents free access to circulating cells and chemical substances. Free virus does not seem to have an important role in the entry of HIV into the brain. However, the contribution of the infection of endothelial cells by HIV needs to be addressed further, as it has been suggested that HIV may use these cells as a reservoir early in infection or as a site of acclimatisation to the brain microenvironment for subsequent entry. ${ }^{4}$ Nevertheless, the prevalent view is that HIV enters the brain inside infected cells of the monocytic lineage; this manner of entry is known as the "Trojan horse hypothesis" (reviewed in Nottet and Gendelman ${ }^{5}$ ). Moreover, circulating HIV infected monocytes are thought to release HIV viral proteins that cause the $\mathrm{BBB}$ to express cell adhesion molecules (CAMs), selectins, and cytokines. ${ }^{6-9}$ These selectins and CAMs can act as "hooks" that cells can latch onto to cross the BBB. Once inside the brain, HIV stimulates the release of chemoattractant cytokines called chemokines. ${ }^{10}$ These, in turn, increase the expression of CAMs on the BBB which can further increase the recruitment of more HIV infected monocytes into the brain. Overall, the tropism of HIV for entry into the brain and the neuronal damage it mediates seems to be correlated with inherent neurovirulent characteristics of HIV. ${ }^{11}$

\section{HIV associated neuropathology}

Up to $90 \%$ of patients with AIDS have a variety of both primary and secondary HIV related brain diseases. Primary brain diseases are attributed directly to the effect of the virus on the brain, and include lymphocytic meningitis, HIV encephalitis (HIVE), HIV leucoencephalopathy (HIVL), vacuolar leucoencephalopathy, and vacuolar myelopathy.
Secondary diseases are a consequence of the immunodeficiency status of the patient and include opportunistic infections (for example, Toxoplasma gondii, cryptococcus, cytomegalovirus, JC virus) and primary non-Hodgkin's B cell lymphomas (reviewed in Budka et al, ${ }^{12}$ Everall and Lantos, ${ }^{13}$ and McArthur ${ }^{14}$ ).

Some of the primary HIV associated brain diseases have similar histological features and are thought to demonstrate a pattern of damage along a pathological continuum. For example, multinucleated giant cells (MGCs) are considered to be a histological hallmark of HIVE but are also found in HIVL. Additionally, varying degrees of myelin pallor, activated macrophage/microglial populations, and neuronal loss can also be prominent. These mixed neuropathological characteristics usually blur our understanding of the course and progression of damage caused by HIV in the brain. However, the main conundrum is how these primary diseases relate to clinical the manifestation of dementia.

The detrimental effect of brain resident HIV seems to depend on increased viral load, neuronal damage, and significant levels of potential neurotoxins within the cerebrospinal fluid (CSF). ${ }^{1516}$ Neuronal loss is generally thought to occur in the symptomatic phase of HIV disease and seems to be prevalent within specific regions and neuronal populations in the brain. Moreover, a consistent $38-50 \%$ neuronal loss in the cortex has been reported in AIDS independent of the clinical presence of dementia. ${ }^{17-19}$ Also, a $30-50 \%$ decrease of large, pyramidal neurons in the cortex and interneurons in the basal ganglia with accompanying simplification of neuritic arbor has been found. ${ }^{2021}$

\section{Putative mechanisms of neuronal damage by HIV}

Recent studies on postmortem brains from patients with AIDS have depicted the cellular distribution of HIV infection within the brain. They found that the brain's resident phagocytic immune cells, macrophages, and micro- 
glia were the major cellular reservoirs of HIV followed by astrocytes and in some cases a very small proportion of neurons. ${ }^{22} 23$ The favoured model of HIV mediated neuronal damage in the brain of patients with AIDS is one of "bystander effect", based on the cellular infectivity model summarised in the figure. Briefly, HIV infected macrophages and microglia are thought to release HIV proteins into the surrounding areas of the brain. These proteins can either promote further HIV replication in infected cells or activate neighbouring cells to produce aberrant levels of cytokines, growth factors, free radicals, and prime the release of neurotoxins (reviewed in Trillo-Pazos and Everall ${ }^{24}$ ). These HIV driven effectors are thought to interact in a complex manner by interfering with intracellular signalling pathways necessary for neuronal viability (for example, decreased growth factor responsiveness, altered neurotransmitter action, aberrant cellular gene activation). These may, in turn, cause subtle changes in neuronal function such as cytoskeletal retraction, upregulation of $\mathrm{Ca}^{2+}$ binding proteins, and inhibition of apoptosis (fig).

HIV infected cells are known to express viral regulatory and structural genes on a temporal basis. ${ }^{25} 26$ Regulatory genes like negative effector (nef) and transactivator (tat) tend to be expressed earlier whereas structural genes like envelope (env) are expressed later in the life cycle of HIV. These genes code for the extracellularly released proteins nef, tat, and glycoprotein of $160 \mathrm{kD}$ (gp 160), respectively. Basic research since the late 1980 s has uncovered a gamut of cytotoxic properties of these HIV proteins. These include:

- Nef protein which has been reported to down regulate viral transcription, increase viral latency and infectivity, and interfere with growth factor mediated signalling pathways involved in proliferation, differentiation, and apoptosis. It has also been shown to be homologous to scorpion toxin. ${ }^{27}$ However, its importance in neuronal damage is only beginning to be addressed.

- Tat protein upregulates viral replication and increases the susceptibility of cells to HIV infection. ${ }^{28}{ }^{29}$ In the brain, tat may both increase the recruitment of HIV infected cells into the CNS, by the upregulation of cytokines and chemokines, ${ }^{78}$ and be neurotoxic by increasing neuronal $\mathrm{Ca}^{2+}$ influx which has been linked to programmed cell death. Moreover, injection of tat into rodents has produced an inflammatory reaction within the brain, astrogliosis, neuronal loss in the grey matter, and increased production of cytokines and nitric oxide synthetase. ${ }^{30}$

- The envelope gp120 glycoprotein is a spliced product of the $e n v$ gene. Its main role is to bind the virion coat to appropriate cell surface receptors of the host cell, including the CD4, B7, and chemokine receptor complex in $T$ cells. Gp120 was initially shown to be neurotoxic by increasing the influx of $\mathrm{Ca}^{2+}$ postsynapti- cally through ionotropic membrane receptors, especially via the n-methyl-Daspartate (NMDA) glutamate receptor. The NMDA receptor seems to have a central role in the formation of memory in the mammalian brain. However, overactivation of this receptor, as found in ischaemia and stroke, produces excitotoxic damage and neuronal death. Moreover, specific antagonists of the NMDA receptor blocks gp120 mediated neurotoxicity both in vitro and in animal models (reviewed in Lipton and Gendelman $\left.{ }^{31}\right)$. Other research has linked gp120 to the production of neurotoxins by macrophages and microglia, ${ }^{32-34}$ the upregulation of cytokines and disruption of neurotransmitter action (reviewed in कै Lipton and Gendelman $^{31}$ ). In vivo $\vec{\circ}$ research, has corroborated gp120 with neuronal damage, astrocytosis, microglial $\vec{\omega}$ changes, and simplification of the dendritic-axonal network (reviewed in Trillo-Pazos and Everall ${ }^{24}$ ).

Apart from putative toxic viral proteins increased levels of cytokines have been consistently reported in the brain and CSF of patients with AIDS. Cytokines are a large family of immunomodulatory proteins comprising interleukins (ILs), chemokines, tumour growth factors (TGFs), tumour necrosis factors (TNFs), and neurotrophic factors. In the brain, they have been found to be both neuroprotective at low levels and potentially neurotoxic at high levels (reviewed in Merril and Benveniste ${ }^{35}$ and Rothwell and Hopkins ${ }^{36}$ ). Cytokines have pivotal roles in conditions of stress, growth, injury, and infection depending on their concentration, site, and duration of action as well as acting synergistically with other molecular effectors like free radicals and other cytokines. They are predominantly released by microglia, macrophages, and astrocytes within the brain constitutively and are upregulated in response to previously described stressors. There is accumulating evidence that there is increased production of potentially damaging cytokines in the brain during AIDS; these include:

- TNF $\alpha$ which is constitutively expressed throughout the brain and can be induced by gp120 and tat. It is involved in the activation of macrophages, general immune response, and in the programmed cell death of activated T cells. ${ }^{35}$ ${ }^{36}$ In AIDS, TNF $\alpha$ has been correlated to astrogliosis, induction of ILs and colony stimulating factors, upregulation of potential neurotoxins like arachidonic acid, platelet activating factor, nitric oxide and the activation of HIV replication via the $\mathrm{NF}-\kappa \mathrm{B}$ transcription factor. TNF $\alpha$ is also thought to cause demyelination within the brain via the demise of oligodendrocytes. It also modulates $\mathrm{Ca}^{2+}$ currents, decreases glutamate uptake by astrocytes, and overstimulates glutamate receptors in neurons (reviewed in Merril and Hopkins ${ }^{35}$ ). 


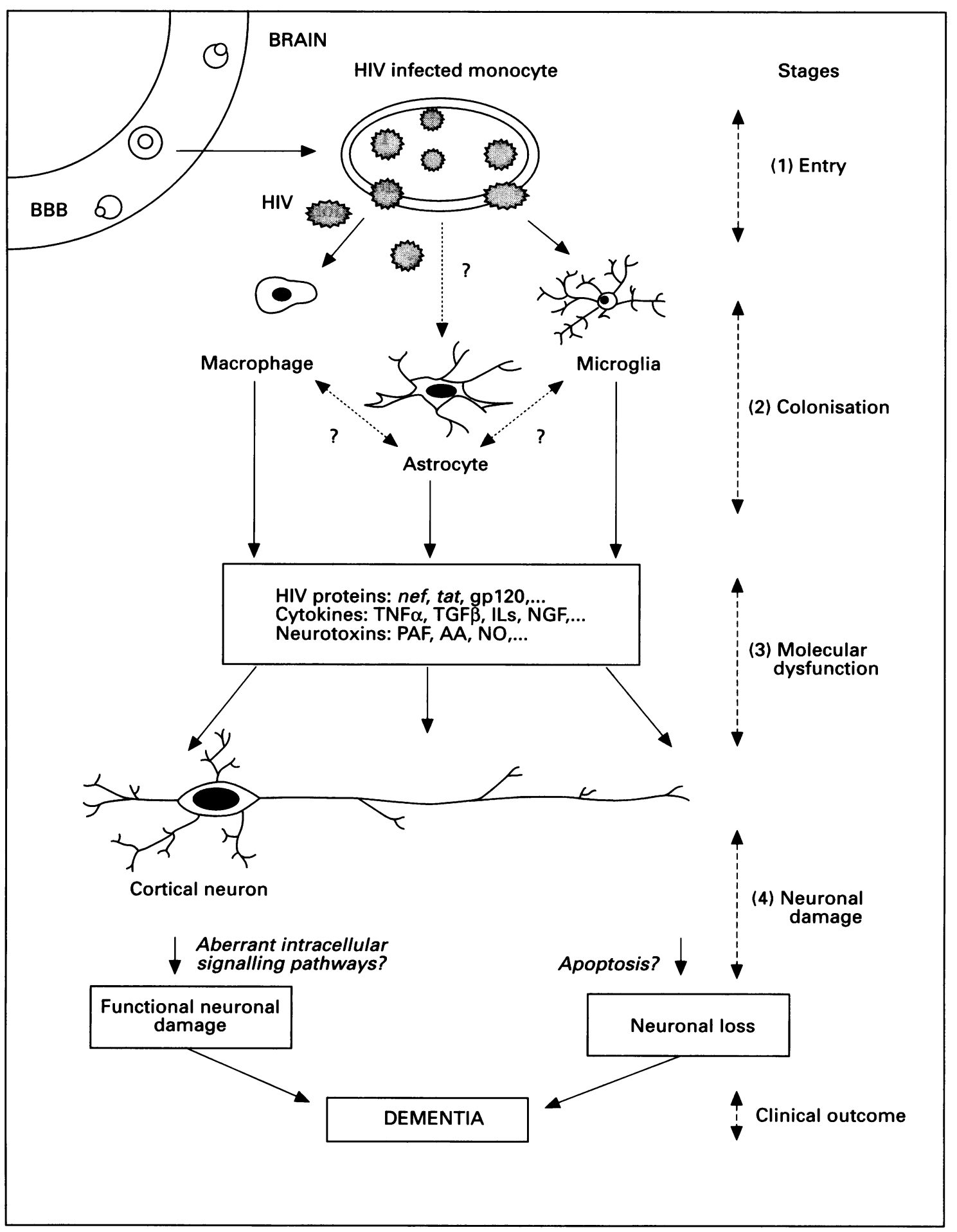

A cellular model of HIV infection in the brain to dementia: a hypothesis.

The stages following HIV entry into the brain can be split into four before the clinical outcome of dementia: (1) HIV entry into the brain is thought to be mediated by activated HIV infected monocyte cells with unregulated surface receptors that attach to the corresponding receptors on endothelial cells in the blood-brain barrier (BBB) (for example, selectins, CAMs), ${ }^{6-9}$ as described in the main text; (2) HIV colonisation of endogenous cells is thought to affect mainly macrophage and microglial cells, but some astrocyte infection by HIV has also been reported. ${ }^{22} 23$ It is at present unclear what the temporal sequence of cellular infection both within the brain and as a function of HIV disease progression is (refer to main text). This colonisation period probably determines what HIV associated primary diseases will be prevalent later on depending on viral and host genotype mediated factors ${ }^{11}$; (3) HIV mediated molecular dysfuntion in the brain sometime after the virus has infected endogenous cells in the brain and has had an unknown period to adjust to its new environment or the actual lymphocyte trafficking of the brain has diminished sufficiently to allow partial or total HIV replication; a cascade of molecular factors are produced ranging from HIV proteins to potential neurotoxins (see main text) (reviewed in Trillo-Pazos and Everall ${ }^{24}$ and Lipton and Gendelman ${ }^{31}$ ). This stage may represent a phase of damage consolidation in HIV associated primary diseases more generally; (4) HIV mediated neuronal damage is thought to occur when the aberrant molecular effectors, whether they be viral or cellular, act either synergistically or indepently to undermine normal cellular function by hijacking intracellular signalling pathways (functional neuronal damage) or inititate the apoptotic pathway in neurons (neuronal loss) (reviewed in Everall et al, ${ }^{16}$ Masliah et al, ${ }^{21}$ and Trillo-Pazos and Everall"4). Dementia may become clinically apparent when the accumulative damage reaches a "critical mass" that undermines the function of neurons in information processing, storage, and retrieval within the human brain. 
- Interleukins are a large family of proinflammatory cytokines that have a multitude of pleotropic roles including initiation of the immune response, activation of lymphocytes, and regulation of body temperature. They can be produced by glial cells, microglia, macrophages, and endothelial cells within the brain. In HIV infection of the brain, IL-1 and IL- 6 have been found at elevated levels. ${ }^{35}$ These ILs have been correlated with astrogliosis, the induction of other cytokines, enhanced HIV replication, and neuronal differentiation.

- TGF $\beta$ is produced by cells of the glial and macrophage lineage within the brain. It curtails the immune response within the brain by inhibiting cytokine production and HIV replication. ${ }^{35}$

So, in the HIV infected brain current evidence suggests a complicated model of neurotoxicity involving both viral and cellular factors culminating in functional neuronal damage and neuronal loss seen in AIDS (reviewed in Trillo-Pazos and Everall ${ }^{24}$ ).

\section{HIV associated dementia (HAD)}

HAD is a progressive impairment of the cognitive, behavioural, and motor abilities of patients with AIDS, resulting in severe dementia and terminal illness within 6 months to a year of onset. ${ }^{36}$ However, this prognosis may well change with the introduction of combination therapies. HAD is usually seen in the late stages of AIDS and does not seem to correspond to any one single HIV primary brain disease. ${ }^{12}$ Moreover, specific neurovirulent HIV strains have been associated with the development of $\mathrm{HAD}^{11}$ but other studies have found no such association. ${ }^{37}$ Recent evidence from postmortem brains of patients with HAD suggests a tentative association between increased viral burden and neuronal damage ${ }^{1522}$ but reports are conflicting. It seems that dendritic, synaptic, and cytoskeletal damage may occur first, followed by cortical pyramidal neuronal loss and then a more generalised loss during the evolution of dementing features. ${ }^{21} 38$ Moreover, increased levels of TNF $\alpha$ messenger $\mathrm{RNA}^{39}$ and inducible nitric oxide synthetase ${ }^{40}$ have been found to correlate with severity of HAD in postmortem brains. Furthermore, as described in this review functional neuronal damage is thought to be orchestrated by a poorly understood complex interaction between HIV viral proteins (for example, gp120, tat, and nef) and cellular products (for example, cytokines, growth factors) (fig). It is imperative to determine how these cited effectors, be they viral or cellular, affect the normal function of neurons to such an extent as to cause the cognitive impairment in patients with AIDS. Firstly, one needs to unravel the ordinary intracellular signalling pathways required for optimal neuronal survival and function within the human brain, and to determine how HIV affects them to such an extent as to undermine their ability to process, store, and retrieve information.
Basic research into the subtle mechanisms of neuronal damage in HIV infection has a crucial role to play if appropriate therapy is to be developed.

G Trillo-Pazos would like to thank the John Ellerman Foundation, Elton John AIDS Foundation, and the Psychiatry Trust for the funds that have made this review and her work possible.

1 An SF, Ciardi A, Giometto B, Scaravilli T, Gray F, Scaravilli F. Investigation on the MHC class II and cytokines and detection of HIV-1 DNA within the brain of asymptomatic and symptomatic HIV-1 positive patients. Acta Neuropathol 1996;91:494-503.

2 Donaldson YK, Bell JE, Ironside JW, Brettle RP, Robertson JR, Busuttil, et al. Redistribution of HIV outside the lymphoid system with onset of AIDS. Lancet 1994;343:382-5.

3 Gray F, Scaravilli F, Everall I, Chretien F, An S, Boche D, t al. Neuropathology of early HIV-1 infection. Brain Pathol 1996;6:1-15.

4 Moses AV, Stenglein SG, Strussenberg JG, Wehrly K, Chesebro B, Nelson JA. Sequences regulating tropism of HIV-1 for brain capillary endothelial cells map to a unique region on the viral genome. F Virol 1996;70 3401-6.

5 Nottet $\mathrm{H}$, Gendelman HE. Unraveling the neuroimmune mechanisms associated HIV-1 associated cognitive/ motor complex. Immunol Today 1995;16:441-8.

6 Hofman FM, Dohadwala MM, Wright AD, Hinton DR Walker SM. Exogenous tat activates CNS derived endothelial cells. $\mathcal{F}$ Neuroimmunol 1994;54:19-28.

7 Lafrenie RM, Wahl LM, Epstein JS, Hewlett IK, Yamada KM, Dhawan S. HIV-1 tat protein promotes chemotaxis and invasive behaviour by monocytes. $₹$ Immunol 1996; and invasive

8 Lafrenie RM, Wahl LM, Epstein JS, Hewlett IK, Yamada KM, Dhawan S. HIV-tat modulates the function of monocytes and alters their interactions with microvesse endothelial cells. A mechanism of HIV pathogenesis. $f$ Immunol 1996;156:1638-45.

9 Nottet HSLM, Persidsky Y, Sasseville VG, Zhai Q-H Bock P, Sharer LR, et al. Mechanisms of transendothelia migration of HIV-1 infected monocytes into the brain. $f$ Immunol 1996;156:1284-95.

10 Schmidtmayerova $H$, Nottet HSL, Nuovo G, Raabe T, Flannagan CR, Dubrovsky L, et al. HIV-1 infection alters chemokine B peptide expression in human monocytes: implications for recruitment of leukocytes into brain and lymph nodes. Proc Natl Acad Sci USA 1996;93:700-4.

11 Power C, McArthur JC, Johnson RT, Griffin DE, Glass JD, Dewey R, et al. Distinct HIV-1 env sequences are associated with neutropism and neurovirulence. Curr Top Microbiol Immunol 1995;202:89-101.

12 Budka H, Wiley CA, Kleihues P, Artigas J, Asbury AK Cho ES, et al. HIV associated disease of the nervous system: review of nomenclature and proposal for neuropathology-based terminology. Brain Pathol 1991;1: 143-52.

13 Everall IP, Lantos PL. The neuropathology of HIV: review of the first 10 years. Int Rev Psych 1991;3:307-20.

14 McArthur JC. Neurologic diseases associated with HIV-1 infection. Curr Opin Infect Dis 1995;8:74-84.

15 Glass JD, Johnson RT. HIV and the brain. Annu Rev Neurosci 1996;19:1-26.

16 Everall IP, Gray F, Masliah E. Neuronal injury and apoptosis. In: Gendelman, Lipton, Epstein, Swindells, eds Neurological and neuropsychiatric manifestations of HIV-I infection. New York: Chapman \& Hall, 1997.

17 Everall IP, Luthert PJ, Lantos PL. Neuronal loss in the frontal cortex in HIV infection. Lancet 1991;337: 1119-21.

18 Everall IP, Gray F, Barnes H, Durigon M, Luthert P, Lantos P. Neuronal loss in symptom-free HIV infection. Lancet 1992;340:1413.

19 Wiley CA, Masliah E, Morey M, Lemere, DeTeresa R Grafe $M$, et al. Neocortical damage during HIV infection. Arafe M, et al. Neocortical dar

20 Masliah E, Ge N, Morey M, De Teresa R, Terry RD, Wiley CA. Cortical dendritic pathology in human immunodeficiency virus encephalitis. Lab Invest 1992;66: 285-91.

21 Masliah E, Ge N, Achim CL, DeTeresa R, Wiley CA Patterns of neurodegeneration. euroAIDS 1996;1 161-73.

22 Bagasra O, Lavi E, Bobroski L, Khalili K, Pestaner JP, Tawadros R, et al. Cellular reservoirs of HIV-1 in the CNS of infected individuals:identification by the combination of in situ polymerase chain reaction and immunohistochemistry. AIDS 1996;10:573-85.

23 Wiley CA, Baldwin M, Achim CL. Expression of HIV reg ulatory and structural mRNA an the CNS. AIDS 1996;10:843-7.

24 Trillo-Pazos G, Everall IP. Neuronal damage and its relation to dementia is acquired immunodeficiency syndrome (AIDS). Pathobiol 1996;64:245-307.

25 Ranki A, Lagerstedt A, Ovod V, Aavik E, Krohn KJ Expression kinetics and subcellular localisation of HIV-1 regulatory proteins Nef, tat and Rev in acutely and regulatory proteins Nef, tat and Rev in acutely and
chronically infected lymphoid cells. Arch Virol 1994; chronically infe $139: 365-78$. 
26 Kim YS, Panganiban AT. Examination of TAR-independent trans activation by HIV-1 tat in human glial cells. $\mathcal{F}$ Neurosci Res 1996;43:652-63.

27 Goldstein G. HIV-1 Tat as a potential AIDS vaccine. Nature Med 1996;1:960-4.

28 Attwood WJ, Berger Jr, Kaderman R, Tornatore CS, Major EO. Human immunodeficiency virus type 1 infection of the brain. Clin Microbiol Rev 1993;6:339-66.

29 Ramazotti E, Vignoli M, Re MC, Furlini G, La Placa M. Enhanced NF- B activation induced by TNF in stably tat-transfected cells is associated with the presence of cell tat-transfected cells is associated with the presence

30 Philippon V, Vellutini C, Gambarelli D, Harkiss G Arbuthnott G, Metzger D, et al. The basic domain of the lentiviral Tat protein is responsible for damage in mouse brain involvement of cytokines. Virology 1994;205: 519-29.

31 Lipton SA, Gendelman HE. Dementia associated with acquired immunodeficiency syndrome. $N$ Engl $f \mathrm{Med}$ 1995;332:934-40.

32 Dawson TM, Dawson VL. Gp120 neurotoxicity in primary cortical cultures. Adv Neuroimmunol 1994;4:167-73.

33 Dawson VL, Dawson TM, Uhl GR, Snyder SH. HIV-1 coat protein neurotoxicity mediated by NO in primary cortical cultures. Proc Natl Acad Sci 1996;90:3256-9.

34 Giulian D, Wendt E, Vaca K, Noonan CA. The envelope glycoprotein of HIV-1 stimulates the release of neurotoxins from monocytes. Proc Natl Acad Sci 1993;90: 2769-2.

35 Merril JE, Benveniste. Cytokines in inflammatory brain lesions: helpful and harmful. TINS 1996;19:331-8.

36 Rothwell NJ, Hopkins SJ. Cytokines and the nervous system 1: actions and mechanisms of action. TINS 1995; 18:130-6.

37 Everall IP. Neuropsychiatric aspects of HIV infection. $f$ Neurol Neurosur Psych 1995;58:399-401.

38 Di-Stefano M, Wilt S, Gray F, Dubois-Dalcq M, Chiodi F. HIV type 1 V3 sequences and the development of dementia during AIDS. AIDS Res Hum Retroviruses 1996;12:471-6.

39 Asare E, Dunn G, Glass J, McArthur J, Luthert P, Lantos P, et al. Neuronal pattern correlates with the severity of HIV associated dementia complex. Am $\mathcal{f}$ Pathol 1996;148: 31-8.

40 Wesselingh SL, Power C, Glass JD, Tyor WR, McArthur JC, Farber JM, et al. Intracerebral cytokine mRNA expression in AIDS dementia. Ann Neurol 1993;33: 576-82.

41 Adamson DC, Wildemann B, Sasaki $M$, Glass JD, McArthur JC, Christov VI, et al. Immunologic NO synthase: elevation in severe AIDS dementia and induction by HIV-1 gp41. Science 1996;274:1917-21. 